\title{
Depth dose calculations
}

\author{
Fatma. Aysun Uğur ${ }^{1, *}$, Elif Gören ${ }^{2}$ \\ ${ }^{1}$ Osmaniye Korkut Ata University, Department of Physics, Osmaniye, Turkey \\ ${ }^{2}$ Cukurova University, Department of Physics, Adana, Turkey
}

\begin{abstract}
In this study, calorimetric method is used to derive the depth doses calculation. In the experiment at the $\mathrm{KeV}$ level, an Ir-192 source with three different gamma energies was used. The source activity is approximately 9.15 curie. The Ir-192 radioactive source is mainly used for industrial purposes. This study had been made to determine organ dose values in any industrial accident. The data are calculated as surface dose and depth dose. Values for different time intervals have been measured. Time and depth parameters for measurements and calculations were obtained for 30 different values. The results of the experiment and the calculations have been compared.
\end{abstract}

\section{Introduction}

Industrial radiography is a frequently used method of non-destructive testing for many industrial products. Industrial radiography is a routine operation requiring high radiation levels and relying primarily on human diligence in observing safety procedures to prevent accidents [1].

Ir-192 source is mostly used in industrial radiography. Ir-192 is normally produced by neutron activation of natural-abundance iridium metal, usually in nuclear reactors. The strength (or specific activity) of a resulting Ir-192 is related to the amount of neutron irradiation and length of time to which the naturalabundance iridium metal is exposed. During irradiation only the stable isotope Ir-191 is activated to produce Ir192 by absorbing a neutron. Radioactive Ir-192 has a half-life of 73.83 days. Radioactive Ir-192 is used principally for non-destructive testing (NDT) and, to a lesser extent, as a radio-tracer in the oil industry. Industrial gamma radiography involves the testing and grading of welds on pressurized piping, pressure vessels, high-capacity storage containers, pipelines, and certain structural welds. Other tested materials include concrete (locating rebar or conduit within the concrete), machined parts, plate metal, and pipe wall. Gamma radiography is also used to identify flaws in metal castings and welded joints, as well as to indicate structural anomalies due to corrosion or mechanical damage [2].

Literature studies have shown that industrial radiography also has various accidents. Workers working with radioactive material in these incidents are exposed to radiation. Experiments are carried out using the calorimetric method to detect these radiation doses. In addition, organ doses taken by people who have been caught in the accident can be calculated by this method. For this purpose, depth dose calculations were made using water phantom and a farmer dosimeter. In this study, Ir-192 was used as a radioactive source. In this study, experiments were carried out in TAEK Cekmece Nuclear Research Centre Health Physics Department SSDL (Secondary Standard Dosimetry Laboratory).

\section{Material and methods}

In the field of ionizing radiations, the absorbed dose is one of the basic quantities commonly used in dosimetry. The main reason of that it is closely related to the biological effects of radiation. Water is of special interest as an absorbing material because it is very similar to human tissue. One of the duties of the national standards laboratories is to establish accurate standards for absorbed dose in water. To this end, much effort has been put into the development of various experimental approaches. One of the most frequently used ones is the calorimetric method because its basic principle is simple [3].

Therefore, the measurements made here were obtained in detail by this method. In these measurements, the values of pressure and temperature were recorded regularly and added to the calculations as correction parameters [1].

\subsection{Depth dose calculations}

The aim of the present work was to measure the depth dose rates by using a water phantom and 192Ir source to obtain experimental and theoretical results. The absorbed dose $\left(D_{d}\right)$ at depths from 0 to $35 \mathrm{~cm}$ in tissue with the source in surface contact was calculated by;

$$
D_{d}=\frac{0,96 A_{2}}{(d+0,24)^{2}} f(d)
$$

Corresponding author: aysunugur@osmaniye.edu.tr 
where, An equal the source activity in unit of curie (A is the exposure rate at $1 \mathrm{~cm}), \Gamma$ is the exposure rate constant, 0.96 is the conversion factor to rads/Roentgen, $\mathrm{d}$ is the tissue depth in unit of $\mathrm{cm}, 0.24$ is the estimated encapsulation thickness in unit of $\mathrm{cm}, \mathrm{f}(\mathrm{d})$ is the scatter and attenuation polynomial of Shalek and Stovall [4] for $\mathrm{d} \leq 15 \mathrm{~cm}$ or $\exp (-0.035 \mathrm{~d})$ for $\mathrm{d} \geq 15 \mathrm{~cm}$.

Since there is a likelihood that the source may have been at a distance of up to $2 \mathrm{~cm}$ from the surface, depth doses were also calculated for these circumstances using standard depth-dose improvement factors with greater distances from the surface. The results are shown in Fig. 3.

\subsection{Experimental Arrangement}

A schematic view of the experimental arrangement is given in Fig. 1. The Ir-192 source is $2 \mathrm{~cm}$ diameter and $0.56 \mathrm{~cm}$ in length. On the day of the experiment the source activity is $9.15 \mathrm{Ci}$. The half-life of the source is 74.2 days. Ir-192 is a meta-stable isotope of Iridium emitting gamma radiation with a low level of Linear Energy Transfer (LET) and high penetration in human body. This isotope frequently has been used for industrial radiography $[5,6]$.

The presence of impurities, especially those with long half-lives and high radiation energies, can significantly alter the absorbed doses. Its energy spectrum is extremely complex, because it has got 30 gammas and $40 \mathrm{X}$-rays [7].

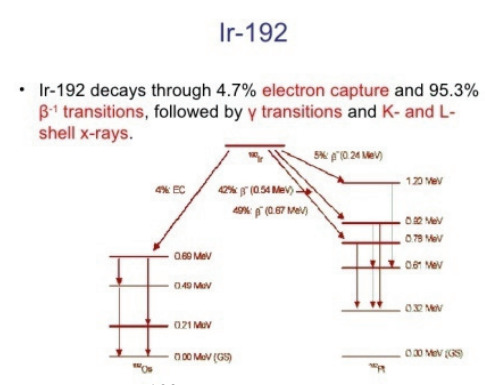

Fig.1. ${ }^{192}$ Ir Decay Scheme

However, for an unshielded source, Table 1 shows the most important energies and frequencies of the emitted gamma and beta particles which have been taken from data compiled by Delacroix et al. [8]. The average energies have been calculated and then added to Table 1 . The distance of the source from the reference plane of measurement is $1 \mathrm{~m}$. In this plane, the beam size is 10 $\mathrm{cm} \times 10 \mathrm{~cm}$; the photon flux at the cross-section border is $50 \%$ of that on the beam axis.

Table 1. Gamma and beta energy distribution for an unshielded ${ }^{192}$ I source [9]

\begin{tabular}{|c|c|c|c|c|}
\hline${ }^{192} \mathbf{I}$ & $\begin{array}{c}\text { Gamma } \\
\mathbf{E}(\mathbf{k e V})\end{array}$ & $\begin{array}{c}\text { Frequency } \\
(\boldsymbol{\%})\end{array}$ & $\begin{array}{c}\text { Beta } \\
\mathbf{E}(\mathbf{k e V})\end{array}$ & $\begin{array}{c}\text { Freuency } \\
(\boldsymbol{\%})\end{array}$ \\
\hline E1 & 317 & 83 & 256 & 6 \\
\hline E2 & 468 & 48 & 536 & 41 \\
\hline E3 & 604 & 8 & 672 & 48 \\
\hline Average & 372 & & 583 & \\
\hline
\end{tabular}

The water phantom consists of a cubic perspex tank, of side $30 \mathrm{~cm}$, with walls $14 \mathrm{~mm}$ thick. The wall facing the beam has a thickness of $4.0 \mathrm{~mm}\left(0.476 \mathrm{~g} . \mathrm{cm}^{-2}\right)$ over a section of $15 \mathrm{~cm} \times 15 \mathrm{~cm}$ to reduce the correction due to the non-equivalence of the perspex front face with water. The phantom, filled with demineralized water, is positioned in such a way that the reference plane is at a depth equivalent to $5 \mathrm{~g} . \mathrm{cm}-2$ in water [9].

The ionization chamber, inserted into a perspex support (1.85 mm thick), can be moved inside the water along the beam axis by means of a translation table fixed on the phantom walls. This allows an accurate adjustment of the chamber position

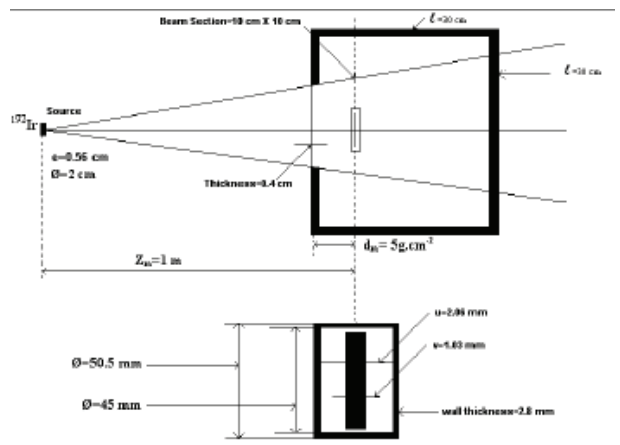

Fig.2. Experimental Arrengement

To avoid long-term variation of the ionization current due to the possible influence of humidity on the ionization chamber, the phantom is filled up and drained every day. The temperature of the chamber is measured with a calibrated thermistor. To match the air conditions of the chamber cavity, the thermistor is placed in air inside a Perspex tube dipped into water. The temperature difference between the positions of the thermistor and of the chamber has been checked and found to be small (less than $0.01^{\circ} \mathrm{C}$ ), so the error introduced is negligible [1].

A Baldwin- Farmer $0.6 \mathrm{cc}$ ionization chamber with a $\pm \% 3$ calibration accuracy (traceable to the National Bureau of standards) was exposed, in air, at a distance of $14 \mathrm{~cm}$ from the source. The positioning error was estimated to be less than $\pm 0.5 \mathrm{~cm}$. This measurement indicated an average exposure rate on 15 June 2016, of $224.06 \mathrm{R} / \mathrm{h}$ at distances of $14 \mathrm{~cm}$. The standard deviation of eight such measurements was less than $\% 3$. This corresponds to a source activity of $9.15 \pm 0.65 \mathrm{Ci}(0.33 \pm$ $0.02 \mathrm{TBq}$ ), with the uncertainty reflecting possible positioning errors. This activity determination was based on an exposure rate constant of $4.8 \mathrm{R}-\mathrm{cm}^{2} / \mathrm{mCi}-\mathrm{h}$ as reported in NCRP (National Council on Radiation Protection and Measurements) Pamplet 40 [7,10]. Although estimates of the exposure-rate constant for ${ }^{192} \mathrm{Ir}$ range from 4.3 to $5.0 \mathrm{R}-\mathrm{cm}^{2} / \mathrm{MCi}-\mathrm{h}$, the value of 4.8 was selected to be consistent with the exposure and dose calculations of this paper which are based on the actual exposure measurements and are independent of the exact source activity $[7,1]$. 


\subsection{Experimental and its calculation}

Firstly, perspex tank has been filled with demineralised water. ${ }^{192} \mathrm{Ir}$ source has been placed at a distance of $3 \mathrm{~cm}$ from the tank. Ionization chamber has been placed at a distance of $2.5 \mathrm{~cm}$. Then, radiation count per minute has been carried out at this position. The exposure unit is generally expressed in unit of Ront/h. However, this expression can be transformed into $\mathrm{rad} / \mathrm{h}$ by multiplying with a factor of $0,96 \mathrm{rad} /$ Ront as given below:

$$
\text { Exposureunit }=\frac{\text { Rönt }}{h} \times 0,96 \frac{\mathrm{rad}}{\text { Rönt }}=\frac{\mathrm{rad}}{h}
$$

Using the proper conversion factors an expression for absorbed dose in the unit of $\mathrm{rad} / \mathrm{h}$ is found as

$$
D=\text { Count }\left(\frac{\text { Rönt }}{h}\right) \times C P x T P x 0,96 \times 60
$$

In this equation; calibration factor of ionization chamber (CP) and temperature-pressure factor (TP as also calculated from equation [11] below) were 1.004 and 1.0165 , respectively

$$
T P=\frac{273,16^{0} K+23,5^{0} C}{293,6} \times \frac{1013}{1008,4}=1,01655
$$

Here, the laboratory temperature on the day of the experiment was taken as $23.5^{\circ} \mathrm{C}$ and pressure as 1008.4 $\mathrm{mb}$. Furthermore, normal temperature and air pressure on sea level were $20^{\circ} \mathrm{C}$ and $1013 \mathrm{mb}$, respectively.

Then the water phantom was irradiated for 20 minutes with a change between SSDL $=100$ and $200 \mathrm{~cm}$ and $d=20-50 \mathrm{~cm}$. The results are shown in Fig. 3 .

\section{Results and discussion}

The purpose of this study was to determine the depth dose. The distance between the radioactive source water phantom is 100 to $200 \mathrm{~cm}$. The depth has been changed from 20 to $50 \mathrm{~cm}$. When the results aren't obtained graphically, the depth-dependent dose change values change exponentially. However, at some points deviations have been observed due to back scattering. When the results are carefully analysed, the changes obtained when the gamma rays emitted from the radioactive source interact with the substance overlap with the classical approach.

Gamma rays interfere with the matter, resulting in photoelectric effect, Compton scattering and pair production (Fig. 4). Fig. 3 is similar to Fig. 5 when viewed.

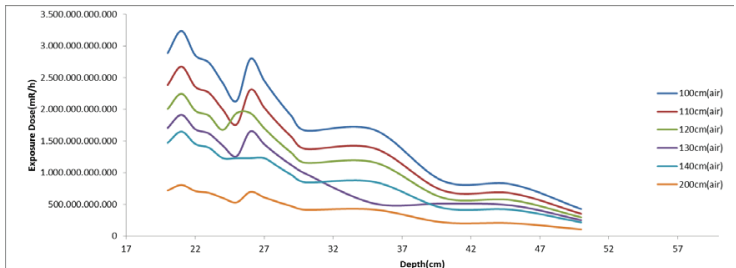

Fig.3. Irradiation dose change for Ir-192 radioactive source

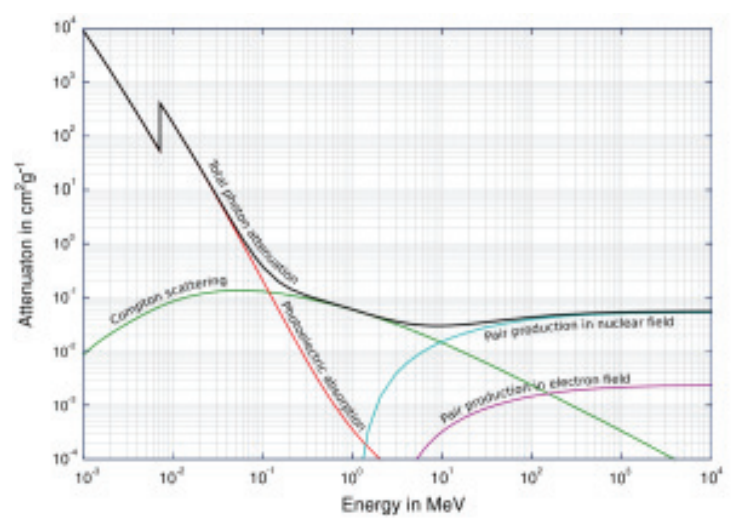

Fig.4. The relative importance of various processes of gamma radiation interactions with matter

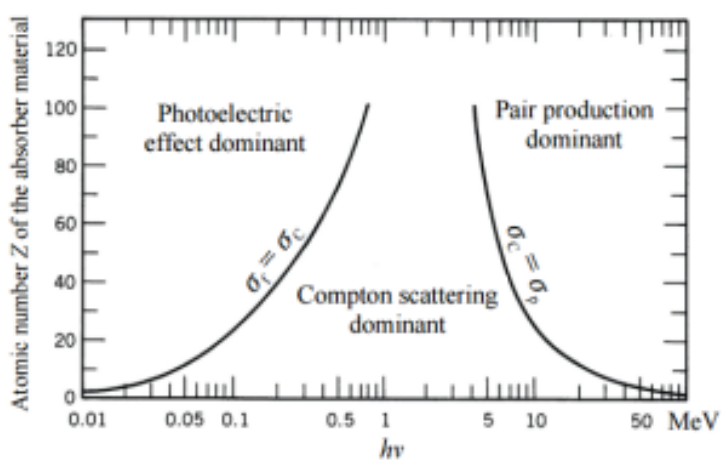

Fig. 5. The relative importance of various processes of gamma radiation interaction with matter.

At low energies, the photoelectric effect and the Compton scattering dominate, while the high energies suppress pair production. (Fig. 5) The linear absorption coefficient for water varies between 0,167 and 0,0706 for gamma rays between $100 \mathrm{KeV}$ and $1000 \mathrm{KeV}$.

\section{Conclusions}

Gamma rays interaction with matter is important from the perspective of shielding against their effect on biological matter. They are considered as ionizing radiation whose scattering by electrons and nuclei leads to the creation of a radiation field containing negative electrons and positive ions. The main modes of interaction of gamma rays with matter are the photo effect both in its photoelectric and photonuclear forms, Compton scattering and electron positron pair production. To a minor extent, photo-fission, Rayleigh scattering and Thomson scattering also occur. Gamma rays have both material attenuation (effect of shielding) and geometric attenuation when interacting with matter. There is also the effect of scattering, which is found in a shielding material and called build up effect. The graph obtained for these reasons is similar to the graph of the interaction of gamma rays with matter. 


\section{References}

1. Uğur et al., Fizika, Volume XVI, Number 2, 516-519 (2010)

2. http://www.isoflex.com/iridium-192

3. M. Boutillon and A.M. Perroche, Phys. Med. Biol., 38 439-454, (1993).

4. R.J. Shalek, M. Stoyall, New York, Academic Press, (1969)

5. A. Jalil, M.A. Molla Health Phys. 62(1): 74-76, (1992)

6. S. Milacic, J. Simic, J. Kobe Med. Sci., 54, 2, E108-E113, (2008)

7. H. F. Eugene and L. B. William, The Medical Basis for Radiation Accident Preparedness, 265-277, (1980)

8. D. Delacroix, J. P. Guerre, P Leblanc, C. Hiykman, Radionuclide and Radiation Protection Data Handbook 2002 Radiat. Prot. Dosim. 98 1-168, (2002)

9. M. Boutillon and A.M. Perroche, Phys. Med. Biol. 38 439-454, (1993)

10. Protection Aganist Radiation From Brachytherapy Sources. NCRP Pamphlet No.40, National Council on Radiation Protection and Measurements, Marh I, (1972)

11. Turkish Atomic Energy Authority website (www.taek.gov.tr) 